\title{
Novel mucus-penetrating liposomes as a potential oral drug delivery system: preparation, in vitro characterization, and enhanced cellular uptake
}

This article was published in the following Dove Press journal:

International Journal of Nanomedicine

| December 201 |

Number of times this article has been viewed

\section{Xiuying $\mathrm{Li}^{\prime}$ \\ Dan Chen' \\ Chaoyi Le ${ }^{2}$ \\ Chunliu Zhu' \\ Yong Gan' \\ Lars Hovgaard ${ }^{3}$ \\ Mingshi Yang ${ }^{4}$ \\ 'Shanghai Institute of Materia \\ Medica, Chinese Academy \\ of Sciences, Shanghai, China; \\ ${ }^{2}$ University of Toronto Mississauga \\ Campus, Ontario, Canada; ${ }^{3}$ Oral \\ Formulation Development, Novo \\ Nordisk A/S, Maalov; ${ }^{4}$ Department \\ of Pharmaceutics and Analytical \\ Chemistry, University of Copenhagen, \\ Copenhagen, Denmark}

Correspondence: Yong Gan Shanghai Institute of Materia Medica, Chinese Academy of Sciences, 50I Haike Road, Shanghai 201203, China

Tel +86 2I $2023 \quad 1000 \quad 1425$

Fax +86 2I $2023 \quad 1000 \quad \mid 425$

Email simm2122@vip.sina.com
Background: The aim of this study was to investigate the intestinal mucus-penetrating properties and intestinal cellular uptake of two types of liposomes modified by Pluronic F127 (PF127).

Methods: The two types of liposomes, ie, PF127-inlaid liposomes and PF127-adsorbed liposomes, were prepared by a thin-film hydration method followed by extrusion, in which coumarin 6 was loaded as a fluorescence marker. A modified Franz diffusion cell mounted with the intestinal mucus of rats was used to study the diffusion characteristics of the two types of PF127 liposomes. Cell uptake studies were conducted in Caco-2 cells and analyzed using confocal laser scanning microcopy as well as flow cytometry.

Results: The diffusion efficiency of the two types of PF127-modified liposomes through intestinal rat mucus was 5-7-fold higher than that of unmodified liposomes. Compared with unmodified liposomes, PF127-inlaid liposomes showed significantly higher cellular uptake of courmarin 6. PF127-adsorbed liposomes showed a lower cellular uptake. Moreover, and interestingly, the two types of PF127-modified liposomes showed different cellular uptake mechanisms in Caco-2 cells.

Conclusion: PF127-inlaid liposomes with improved intestinal mucus-penetrating ability and enhanced cellular uptake might be a potential carrier candidate for oral drug delivery.

Keywords: Pluronic F127, mucus-penetrating, particles, liposomes, oral drug delivery

\section{Introduction}

Liposomal formulations have for many years been among the most promising nanocarriers for drug delivery and have been widely studied for many years. ${ }^{1-5}$ These vesicular carriers composed of one or more phospholipid bilayers are able to carry both hydrophilic and hydrophobic drug molecules due to their amphiphilic nature. They are easily biodegradable as well as biocompatible, and have strong similarities in composition to a biomembrane. However, liposomes are not as yet an ideal vehicle for oral drug delivery. One of the limitations of this system lies in the poor mucuspenetrating properties of liposomes. ${ }^{6,7}$ Owing to the hydrophobic properties of liposomes, they can be easily trapped or wiped by the mucus blanket, which greatly compromises their application in oral drug delivery.

Intestinal mucus has a thickness of 100-800 $\mu \mathrm{m}$ and is composed of a complex network of highly branched glycoproteins, lipid, cellular and serum macromolecules, electrolytes, cells, and other cellular debris. ${ }^{8,9}$ The high viscoelasticity and adhesion of mucus, along with an abundance of possible particle-environment interactions, can severely retard the diffusion of nanocarriers or macromolecules through the mucus layer. ${ }^{10,11}$ Nanocarriers or macromolecules that do not efficiently penetrate the deeper 
mucus layers and thereby reach the underlying epithelium will be eliminated by mucus clearance mechanisms. Enhancing mucus-penetrating properties is considered to be of great importance in facilitating prolonged retention and more uniform distribution of drug carriers at mucosal surfaces. ${ }^{9,12,13}$ However, in order to improve cellular uptake and permeability in the epithelium, nanocarriers must penetrate mucus, which is a longstanding challenge in mucous membrane drug delivery.

It has recently been reported that polystyrene and polylactic-co-glycolic acid (PLGA) nanoparticles sufficiently coated with certain Pluronic ${ }^{\circledR}$ polymers or polyethylene glycol show lower degrees of mucoadhesion. This was proposed on the basis of a reduction of hydrophobic and/or electrostatic interactions, allowing for rapid penetration of the nanoparticles through human mucus. ${ }^{14-18}$ However, almost all of the studies of mucus-penetrating particles are focused on lung or cervicovaginal mucus, and very often use nonbiodegradable nanoparticles as drug delivery carriers. To the best of our knowledge, no mucus-penetrating liposomal formulation has been reported so far.

The aim of this study was to investigate the functionality of mucus-penetrating liposome formulations and explore their intestinal cellular uptake mechanism. Pluronic F127 (PF127) was chosen as a liposomal modifier. Two types of PF127-modified liposomes ie, PF127-inlaid and PF127adsorbed, were designed. The mucus-penetrating properties of the PF127-modified liposomes were studied using a modified Franz diffusion cell. Cellular uptake studies of the two types of PF127-modified liposomes were conducted in Caco-2 cells and the uptake mechanism of the two types of PF127-modified liposomes was also investigated.

\section{Materials and methods Materials}

Egg phosphatidylcholine (EPC) was purchased from QP Corporation (Tokyo, Japan). PF127 was kindly donated by BASF (Ludwigshafen, Germany). RPMI 1640 medium and 0.25\% trypsin/0.53 mmol/L EDTA were purchased from Invitrogen (Ontario, CA). Fetal bovine serum was obtained from Sijiqing Biological Engineering Materials Co Ltd (Zhejiang, China). Paraformaldehyde was purchased from Sinopharm Chemical Reagent Co Ltd (Shanghai, China). Penicillin, streptomycin, and 2-(4-amidinophenyl)-6-indolecarbamidine dihydrochloride (DAPI) were purchased from Beyotime Institute of Biotechnology (Jiangsu, China). Lovastatin was purchased from Ruibang Laboratories (Zhejiang, China). Chlorpromazine was a generous gift from Grand Pharmaceutical Co Ltd
(Wuhan, China). Methyl- $\beta$-cyclodextrin, filipin, cytochalasin D, coumarin 6, and Hank's balanced salt solution (HBSS) were all purchased from Sigma-Aldrich (Saint Louis, MO). All other chemicals were of analytical reagent grade.

\section{Preparation of liposomal carriers}

Liposomes were prepared by the thin film hydration technique. Two methods were used to incorporate PF127 into the liposomes. ${ }^{19}$ Using the first method, the liposomal carrier was prepared by drying a chloroform solution containing both lipid and PF127. Hydration of the film was then performed in distilled water for one hour at $25^{\circ} \mathrm{C}$ to a final lipid concentration of $5 \mathrm{mg} / \mathrm{mL}$. The multilamellar vesicles were then extruded six times through $200 \mathrm{~nm}$ polycarbonate membranes (Avestin Inc, Canada). In the second method, liposomes were prepared as described earlier without addition of PF127. However, this was added after extrusion of the formed liposomes for absorption onto their surfaces.

In order to optimize the ratio of PF127 to EPC, the effect of different amounts of PF127 on the particle size and zeta potential of the preformed liposomes was studied. Fluorescence labeling of the liposomal carriers was performed using hydrophobic coumarin 6 during the preparation of thin lipid films. The marker was dissolved together with EPC in chloroform, and the liposomal carriers were prepared as described above.

\section{Characterization of liposomal carriers}

The particle size, size distribution, and zeta potential of the liposomal carriers were determined at room temperature in distilled water using a Malvern Zetasizer NanoZS (Malvern Instruments, Worcestershire, UK). The morphology of the unmodified liposomes, PF127-inlaid liposomes, and PF127-adsorbed liposomes was analyzed using a transmission electron microscope (Philips CM200, Eindhoven, The Netherlands) at an accelerating voltage of $160 \mathrm{kV}$. Samples were deposited on carbon-coated copper grids and stained with phosphotungstic acid $(1 \% \mathrm{w} / \mathrm{v}) .{ }^{20}$ To study the effect of temperature on the physicochemical properties of the liposomes, the solutions were aliquoted into two vials and stored for 2 hours at $4^{\circ} \mathrm{C}$ and $37^{\circ} \mathrm{C}$, respectively. The particle sizes of the liposomes were determined using the Malvern Zetasizer NanoZS before and after incubation.

\section{Detergent-resistant membrane level}

The turbidity of the liposome samples with or without PF127 modification exposed to Triton ${ }^{\circledR} \mathrm{X}-100$ was measured as absorbance by ultraviolet spectrophotometry at $400 \mathrm{~nm}$ and 
room temperature using a $4 \mathrm{~cm}$ light-path cell. Samples were occasionally stirred to ensure uniform mixing. The turbidity measurements were normalized to the control turbidity value of the liposome suspensions before treatment with Triton X-100. The effect of various doses of Triton X-100 on lipid turbidity was studied.

\section{Collection and preparation of native mucus samples}

Native rat intestinal mucus was collected for the mucuspenetrating study. ${ }^{21}$ Male Wistar rats which had been fasted overnight were sacrificed. The intestines were isolated and cut open, and all contents were removed by rinsing with cold saline. The mucus was then gently scraped with a cover slide and aliquoted in small glass/Eppendorf vials. The mucus collected was stored at $-80^{\circ} \mathrm{C}$ until use. Prior to the mucus penetration experiments, the frozen sample vials were thawed over approximately 15 minutes to $37^{\circ} \mathrm{C}$. All experiments were performed according to the regulations of the Animal Care and Use Committee of Shanghai Institute of Materia Medica.

\section{In vitro mucus penetration study}

The mucus ( $350 \mathrm{mg}$ ) was equilibrated in a constant temperature culture vibrator at $37^{\circ} \mathrm{C}$ for 15 minutes at a speed of 100 rpm to ensure homogeneous dispersion of mucus. ${ }^{22,23}$ It was then placed in the donor chamber of a modified Franz diffusion cell with a polycarbonate membrane (pore size $2 \mu \mathrm{m}$ ) located between the donor and receptor chambers to support the mucus. Then $50 \mu \mathrm{L}$ of coumarin 6-loaded unmodified liposome, PF127-inlaid liposome, or PF127-adsorbed liposome solutions were added onto the surface of the mucus and equilibrated for another 15 minutes in the vibrator under the same conditions. The receptor chamber was filled with phosphate-buffered saline. At fixed time intervals, $1 \mathrm{~mL}$ samples from the receptor chamber were withdrawn and replaced with the same volume of prewarmed fresh phosphate-buffered saline. The samples were subsequently treated with Triton X-100 to lyse the liposomes and extract coumarin 6 . The concentration of coumarin 6 was determined using a microplate reader.

The apparent permeability coefficient $\left(\mathrm{P}_{\text {app }}\right)$ was calculated using the following equation:

$$
\mathrm{P}_{\text {app }}=\mathrm{dQ} / \mathrm{dt} \times 1 / \mathrm{AC}_{0}
$$

where $\mathrm{dQ} / \mathrm{dt}$ is the flux of coumarin 6 through the intestinal mucus; $\mathrm{C}_{0}$ is the initial concentration of the coumarin 6 in the donor chamber; and $\mathrm{A}$ is the area of the membrane between the donor chamber and the receptor chamber. The permeation enhancement ratio $(\mathrm{R})$ was calculated by dividing the $\mathrm{P}_{\text {app }}$ of PF127-modified liposomes by that of unmodified liposomes.

\section{Cell culture}

Caco-2 cell lines were obtained from the American Type Culture Collection (ATCC, Manassas, VA), and the cells of passages $30-40$ were used in this study. The cells were maintained in RPMI 1640 medium supplemented with 10\% heat-inactivated fetal bovine serum, $1 \%$ penicillin, and $1 \%$ streptomycin at $37^{\circ} \mathrm{C}$ in $5 \% \mathrm{CO}_{2} / 95 \%$ air atmosphere in a humidified incubator. The cells were placed in a $75 \mathrm{~cm}^{2}$ flask at a density of $1 \times 10^{6}$ cells/flask. The medium was changed every 2-3 days and the cells were then harvested at $80 \%$ confluence with trypsin-EDTA and passaged.

\section{Cellular uptake study}

Confocal laser scanning microcopy (CLSM) and flow cytometry (FACS) were used to assess intracellular uptake efficiency. For the CLSM analysis, the Caco-2 cells were seeded on glass cover slips in 24-well plates at a density of $1 \times 10^{5}$ cells $/ \mathrm{cm}^{2}$. After culturing for 24 hours, the culture medium was discarded and the cells were washed three times using HBSS. After $1 \mathrm{~mL}$ of liposomal formulations containing coumarin $61 \mu \mathrm{g} / \mathrm{mL}$ was added, the cells were incubated for 2 hours at $37^{\circ} \mathrm{C}$. The sample solutions were then discarded and the cells were washed three times with HBSS. The cells were fixed with $4 \%$ paraformaldehyde solution and the cell nuclei were stained using DAPI. The samples were embedded in phosphate-buffered saline/glycerol (1:1) and examined under an inverted confocal microscope (FV1000, Olympus, Tokyo, Japan). The coumarin 6 and DAPI were investigated at $488 / 520 \mathrm{~nm}$ and $364 / 460 \mathrm{~nm}$ (excitation/ emission), respectively.

For FACS analysis, the cells were seeded in 12-well culture plates at a density of $1 \times 10^{5}$ cells $/ \mathrm{cm}^{2}$. After 2 hours of incubation with the liposomal solutions, the cells were washed three times with HBSS and trypsinized for 2 minutes with $0.25 \%$ trypsin/ $0.53 \mathrm{mmol} / \mathrm{L}$ EDTA. Trypsinization was terminated by adding cold culture medium. The cells were detached gently by pipetting. After centrifugation, the treated samples were washed three times with HBSS and then transferred to FACS tubes. The cells were analyzed by FACS using BD FACS Calibur fluorescent-activated flow cytometry and BD CellQuest software. This experiment was performed in triplicate and a total of 20,000 cells per sample were analyzed. 


\section{Cellular association and uptake mechanism}

In order to identify the endocytotic mechanisms of PF127modified liposomes, cellular uptake studies were performed in the presence of specific inhibitory agents for different types of endocytosis. Caco-2 cells were first incubated in HBSS containing the specific inhibitory agents for one hour at $37^{\circ} \mathrm{C}$, and subsequently exposed to the liposomal solutions for 2 hours at $37^{\circ} \mathrm{C}$. Control experiments were performed in HBSS alone followed by cellular uptake studies for the different liposomes.

The effect of low temperature on the Caco-2 association with different liposomal nanocarriers was investigated at $4{ }^{\circ} \mathrm{C}$. Methyl- $\beta$-cyclodextrin $10 \mathrm{mmol} / \mathrm{L}$ in the presence of lovastatin $1 \mu \mathrm{g} / \mathrm{mL}$ was used to deplete cholesterol, ${ }^{24}$ which had been proved to inhibit caveolae-independent and clathrin-independent endocytosis. Filipin $1 \mu \mathrm{g} / \mathrm{mL}$ was used to inhibit caveolae-mediated or caveolae-like domainmediated endocytosis, while chlorpromazine $10 \mu \mathrm{g} / \mathrm{mL}$ inhibited clathrin-mediated endocytosis. ${ }^{25}$ Inhibition of macropinocytosis was achieved by incubation of cells with cytochalasin D $1 \mu \mathrm{g} / \mathrm{mL} .{ }^{26}$

\section{Results}

\section{Preparation and characterization of liposomal nanocarriers}

Liposomal nanocarriers were obtained by the thin film hydration technique, as described in the Materials and methods section.
Figure 1 shows the particle size and zeta potential of liposomes in the presence of PF127 at molar ratios of 0:100, 1:100, 2.5:100, 5:100, 10:100, and 15:100. The presence of PF127 neutralized the negative surface charge of the liposomes. The particle size of the liposomes increased with an increase in the amount of PF127. A molar ratio of 5\% was chosen to ensure a highly dense coating and partial surface charge neutralization of the liposomal surface with PF127 in order to improve the mucus-penetrating properties. However, this coating did not significantly change the particle size of the liposomes.

As shown in Table 1, unmodified liposomes had an average hydrodynamic diameter of $191.7 \pm 11.2 \mathrm{~nm}$ with a polydispersity of $0.195 \pm 0.011$ and a negative zeta potential of approximately $-8.9 \mathrm{mV}$. The incorporation of PF127 in the liposomes prepared by the two different methods did not have a distinct effect on particle size, but led to a significant $(P<0.05)$ decrease in the zeta potential, which was reduced to $-4.1 \mathrm{mV}$ (PF127-inlaid) and $-3.5 \mathrm{mV}$ (PF127adsorbed).

As shown in Figure 2A, similar spherical morphologies were observed for unmodified liposomes, PF127-inlaid liposomes, and PF127-adsorbed liposomes, which indicates that PF127 did not alter the morphology of the liposomal nanocarriers.

It was necessary to study the effect of temperature on the physical properties of liposomes due to the thermosensitive properties of PF127. The particle size change in

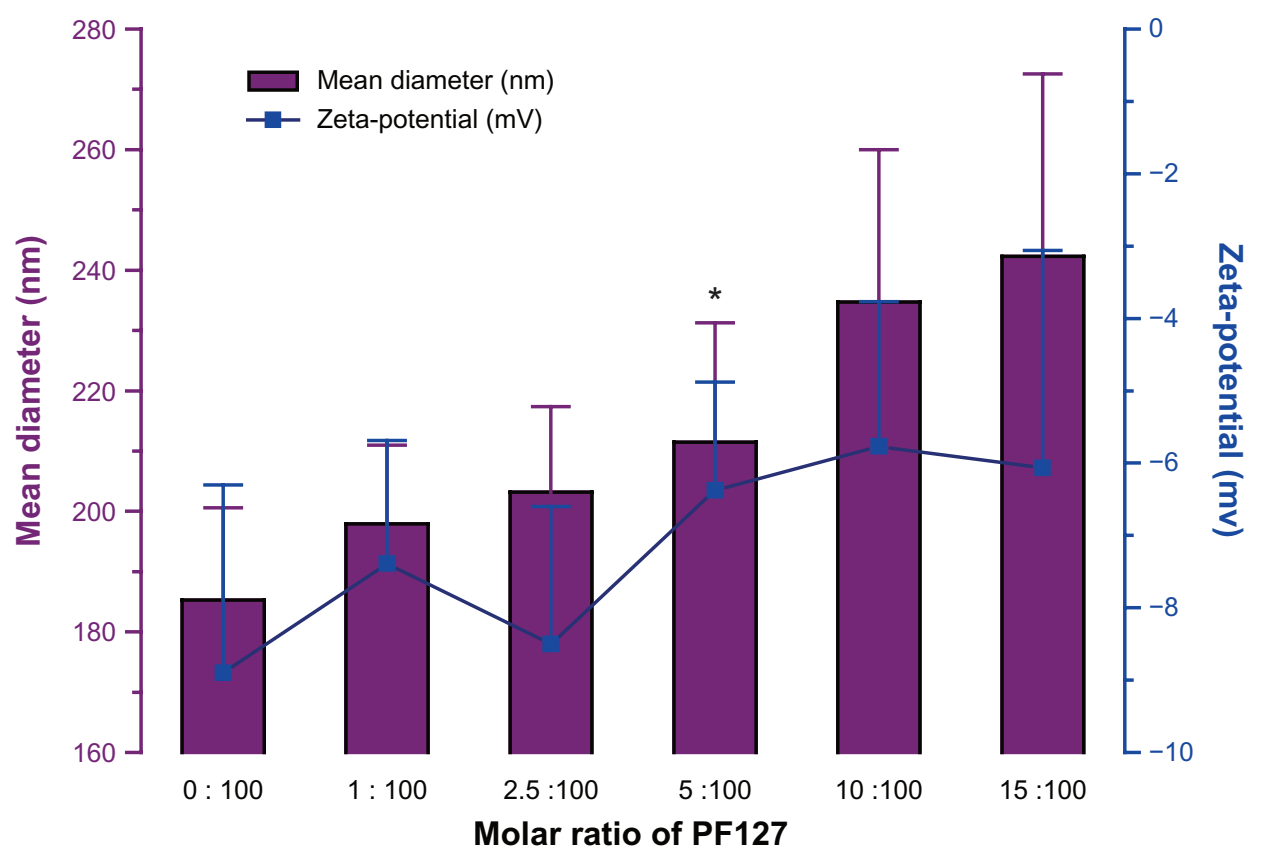

Figure I Particle size and zeta potential of liposomes with different amounts of Pluronic ${ }^{\circledR}$. 
Table I Particle size, polydispersity, and zeta potential of unmodified liposomes, Pluronic ${ }^{\circledR}$ PFI27-inlaid liposomes, and Pluronic PFI27-adsorbed liposomes

\begin{tabular}{llll}
\hline Formulation & $\begin{array}{l}\text { Mean diameter } \\
(\mathbf{n m})\end{array}$ & \multicolumn{1}{l}{$\begin{array}{l}\text { Zeta-potential } \\
(\mathbf{m V})\end{array}$} \\
\hline Unmodified liposomes & $191.7 \pm 11.2$ & $0.195 \pm 0.011$ & $-8.93 \pm 1.67$ \\
PFI27-inlaid liposomes & $185.6 \pm 8.9$ & $0.103 \pm 0.005$ & $-4.12 \pm 2.32$ \\
PFI27-adsorbed & $203.2 \pm 13.7$ & $0.206 \pm 0.034$ & $-3.54 \pm 1.37$ \\
liposomes & & & \\
\hline
\end{tabular}

Note: Data were shown as mean \pm standard deviation $(n=3)$.

Abbreviation: PI, polydispersity index.

the liposomes after incubation at $4^{\circ} \mathrm{C}$ and $37^{\circ} \mathrm{C}$ for 2 hours is shown in Figure 2B. These results show that although modified with the same thermosensitive polymer, the two PF127-modified liposomes had different thermosensitive properties. When kept at $4^{\circ} \mathrm{C}$ for 2 hours, the particle size of the PF127-inlaid liposomes increased from $185 \mathrm{~nm}$ to $227 \mathrm{~nm}$, whereas the PF127-adsorbed liposomes converted into aggregates at a micrometer level. Liposomal aggregation was clearly observed in both PF127-inlaid liposomes and PF127-absorbed liposomes after 12 hours of incubation at $4{ }^{\circ} \mathrm{C}$. However, none of the PF127-modified liposomes changed significantly at $37^{\circ} \mathrm{C}$. The results indicated that the thermosensitive properties of PF127 were partially compromised when incorporated into liposomes during the preparation period, while the construction of PF127-adsorbed liposomes at low temperature retained thermosensitivity.

\section{Detergent-resistant membrane level altered by PFI 27}

The ability of PF127 to alter detergent resistance at the membrane level in the mixed liposome was evaluated, given that the ability to resist detergent extraction is a characteristic of formation of dense gel/ordered domains in the liposome. ${ }^{27}$ Incorporation of PF127 into liposomes caused a significant shift of the turbidity curve in the presence of Triton X-100. A slower decrease in turbidity was observed for PF127-inlaid liposomes and PF127-adsorbed liposomes (Figure 2C), which indicated that PF127 was able to protect domain lipids from being solubilized by Triton X-100. The effect was more significant for PF127-inlaid liposomes, which suggested that PF127-inlaid liposomes caused highly ordered and tightly packed domains compared with unmodified liposomes and PF127-adsorbed liposomes.
A
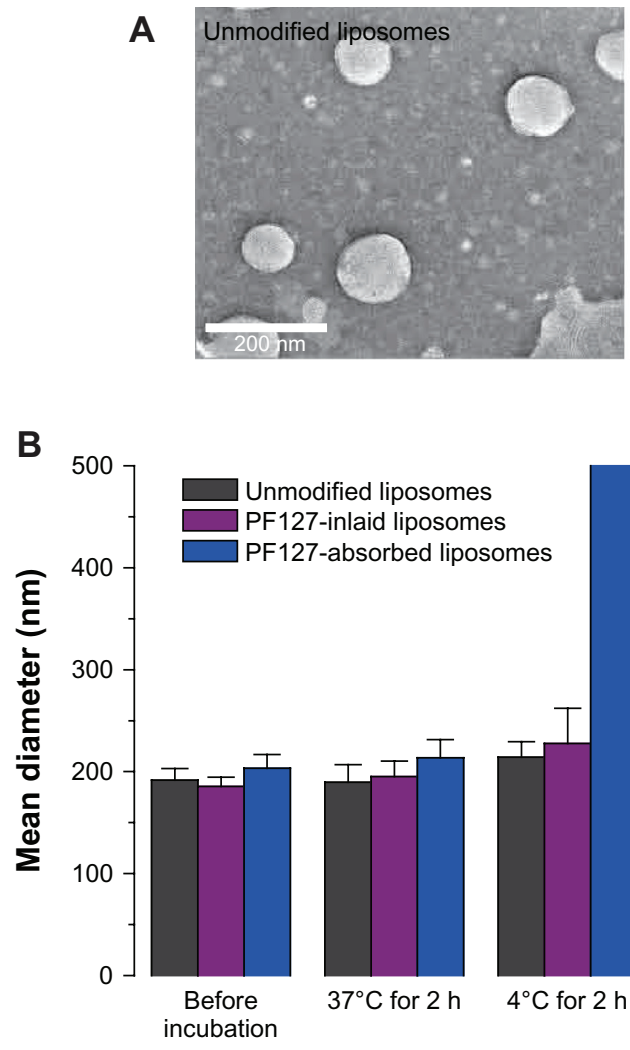
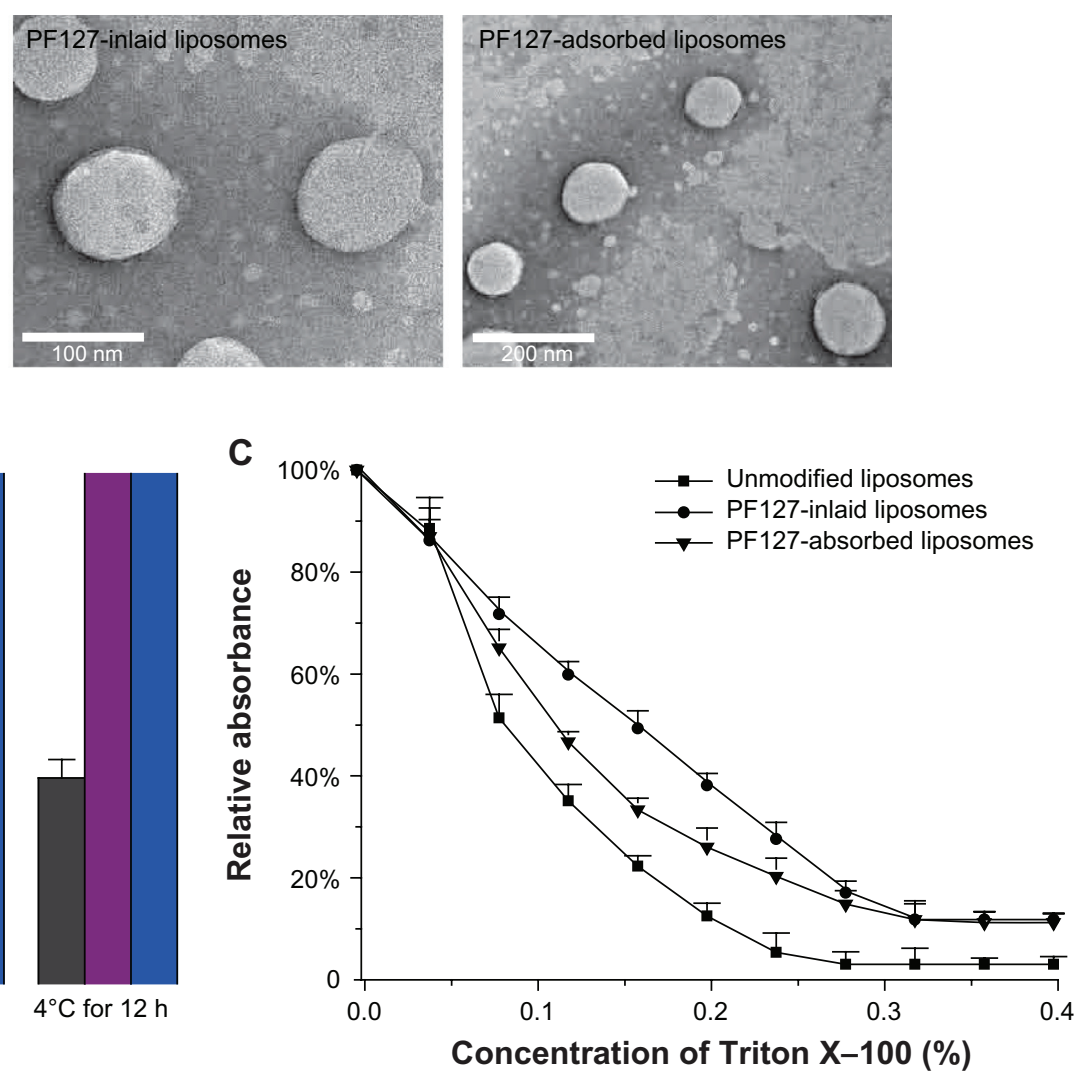

Figure 2 (A) Transmission electron micrographs of unmodified liposomes, Pluronic ${ }^{\circledR}$ FI27-inlaid liposomes and Pluronic FI27-adsorbed liposomes. (B) Particle size change in liposomes after incubation at $37^{\circ} \mathrm{C}$ for 2 hours and $4^{\circ} \mathrm{C}$ for 2 hours or 12 hours. (C) Influence of Triton X-100 on relative absorbance at 400 nm of unmodified liposomes $(\bullet)$, PFI27-inlaid liposomes $(\bullet)$, and PFI27-adsorbed liposomes ( $)$. 


\section{In vitro intestinal mucus-penetrating studies}

The cumulative amounts of coumarin 6-loaded liposomal carriers penetrating through the mucus layer are shown in Figure 3. Comparing unmodified liposomes with the two types of PF127-modified liposomes, an enhanced permeability of coumarin 6 was found for modified liposomes. Due to the hydrophobic properties of coumarin 6 , the association between the marker and liposomal formulations was quite strong. The entrapment efficiency of coumarin 6 in the liposomal carriers was measured to be approximately $100 \%$, and less than $2.0 \%$ of coumarin 6 was released from the liposomal carriers after 4 hours of incubation at $37^{\circ} \mathrm{C}$ in phosphatebuffered saline (data not shown). Therefore, it is reasonable to assume that coumarin 6 was cotransported with the liposomal nanocarriers, ${ }^{16}$ and thus it is acceptable to use coumarin 6 as a marker of liposomal transport in the Franz diffusion cell. The cumulatively transported amounts of coumarin 6 in the two types of PF127-modified liposomes were increased by more than six-fold compared with unmodified liposomes (Figure 3B). As a control, permeability of coumarin 6-loaded unmodified liposomes was investigated in the absence of rat intestinal mucus in the donor chamber of the Franz diffusion cell. As shown in Figure 3B, more than 95\% of coumarin 6 penetrated through the polycarbonate membrane in this case. This indicates that native rat intestinal mucus has the barrier properties of a liposomal formulation.

The $\mathrm{P}_{\text {app }}$ of coumarin 6-loaded liposomal carriers and the permeation enhancement ratio of the two types of PF127modified liposomes are presented in Table 2. The results show that the two types of PF127-modified liposomes increased permeation of coumarin 6 as compared with unmodified liposomes. The $\mathrm{P}_{\text {app }}$ of coumarin 6-loaded PF127inlaid liposomes and PF127-adsorbed liposomes was almost 5-7-fold higher than that of unmodified liposomes. However, there was no significant difference in $\mathrm{P}_{\text {app }}$ observed between PF127-inlaid liposomes and PF127-adsorbed liposomes. This indicates that PF127 improved the mucus-penetrating properties regardless of the type of incorporation between polymer and liposomes.

\section{Cellular uptake study in Caco-2 cells}

Caco-2 uptake of the liposomal carriers was visualized using CLSM. As shown in Figure 4 (left), the Caco-2 cellular uptake efficiency of coumarin 6 was influenced by the type of PF127 modification in the liposomes. The PF127-inlaid liposomes showed a higher association efficiency with the cells than did the unmodified liposomes. Stronger green fluorescence was observed to be associated with Caco-2 cells in the PF127-inlaid liposome group compared with the unmodified liposome group, whereas the cellular uptake of liposomes was decreased when PF127 was simply adsorbed onto the surface of the liposomes. The results from FACS, as shown in Figure 4 (right), strongly support the findings of the CLSM study. For the PF127-inlaid liposomes, the cellular association efficiency was enhanced by approximately 1.5-fold compared with unmodified liposomes, whereas the cellular association efficiency was decreased to less than $70 \%$ when PF127 was merely attached to the surface of the liposomes by adsorption.

As mentioned above, the release of coumarin 6 from the liposomal formulation after 4 hours of incubation at $37^{\circ} \mathrm{C}$ in phosphate-buffered saline was usually less than $2.0 \%$ due to its hydrophobic properties. Therefore, the contribution of
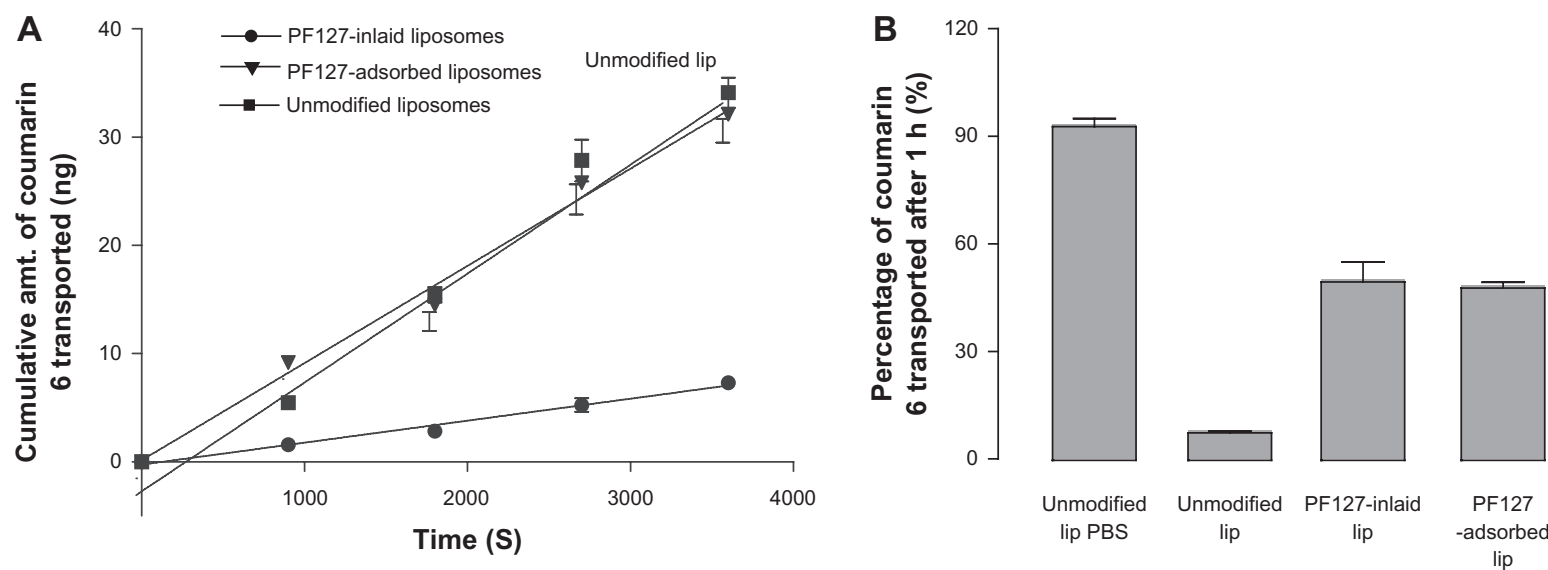

Figure 3 (A) Cumulative amounts of coumarin 6 in liposomes transported through intestinal mucus as a function of time. Unmodified liposomes ( $\mathbf{\square}$ ), Pluronic ${ }^{\circledR}$ FI27-inlaid liposomes $(\bullet)$, Pluronic FI27-adsorbed liposomes ( $\boldsymbol{\nabla})$. (B) Percentage of cumulative amounts of coumarin 6 loaded in liposomal formulations transported through intestinal mucus layer after one hour ( $\mathrm{n}=3$; data shown as the mean \pm standard deviation).

Abbreviations: lip, unmodified liposomes; PBS, phosphate-buffered solution. 
Table 2 Permeability value for coumarin 6-loaded unmodified liposomes, Pluronic ${ }^{\circledR}$ PFI27-inlaid liposomes and Pluronic PFI27adsorbed liposomes transported through intestinal mucus

\begin{tabular}{lcl}
\hline Formulation & $\mathbf{P}_{\text {app }}\left(\times 10^{-7} \mathbf{c m} / \mathbf{s}\right)$ & $\mathbf{R}$ \\
\hline Unmodified liposome & $1.88 \pm 0.23$ & - \\
PFI27-inlaid liposomes & $12.37 \pm 1.05$ & $6.58 \pm 0.56$ \\
PFI27-adsorbed liposomes & $11.64 \pm 1.16$ & $5.84 \pm 0.62$
\end{tabular}

Notes: Data shown as mean \pm standard deviations $(n=3)$.

Abbreviations: $P_{\text {app, }}$, the apparent permeability coefficient; $R$, permeation enhancement ratio.

free coumarin 6 to the total cellular association of the carriers was negligible. In the Caco-2 cell uptake study, coumarin 6 detected inside the cells could have been derived from cellular association of the liposomal carriers rather than the free marker released from the liposomal formulations. These findings indicate that the mechanism of cellular uptake for these two types of PF127-modified liposomes was different, even though both formulations improved the mucus-penetrating properties relative to unmodified liposomes.

\section{Uptake mechanism of liposomal carriers in Caco-2 cells}

The cellular uptake mechanism of the two types of PF127modified liposomes were investigated using different inhibiting agents and different temperatures. The effect of low temperature on the Caco-2 association with the different liposomal carriers was evaluated by incubating the different formulations at $4^{\circ} \mathrm{C}$. The same liposomal nanocarriers treated with HBSS for one hour were used as the control group. As shown in Figure 5, a significant reduction in Caco- 2 cell association efficiency was observed at $4^{\circ} \mathrm{C}$ for all three formulations as compared with that at physiological temperature. The cellular association of PF127-inlaid liposomes and PF127-adsorbed liposomes decreased significantly $(P<0.01)$ to $20 \%$ and $35 \%$, respectively, while the unmodified liposomes still had a cellular association of about $50 \%$ as compared with the control group $(P<0.05)$. These results indicate that the cellular uptake of PF127-inlaid liposomes and PF127-adsorbed liposomes relied more on an energydependent pathway than did the unmodified liposomes.

The effect of nonspecific metabolic inhibitors on the cellular association of the liposomes was also investigated. It was reported that liposomes could be internalized into cells by different mechanisms, including clathrin-dependent endocytosis, caveolae-dependent endocytosis, macrocytosis, and fusion, according to the type of cell, composition, surface charge, and size of the liposome. ${ }^{25,28}$ In our study, the cellular association of the unmodified liposomes was significantly influenced by filipin, with a reduction in cellular association of about $50 \%$ (Figure $5 \mathrm{~A}$ ), while the effects of methyl- $\beta$ cyclodextrin/lovastatin, chlorpromazine, and cytochalasin $\mathrm{D}$ on the cellular association of unmodified liposomes was negligible. This indicates that in addition to pathways that are not energy-dependent, caveolae-mediated endocytosis is also involved in the uptake of unmodified liposomes by Caco-2 cells. Preincubation of Caco-2 cells with filipin $1 \mu \mathrm{g} / \mathrm{mL}$ resulted in significant reduction of the cellular association of PF127-inlaid liposomes (approximately 50\%). However, it did not completely inhibit active uptake of the liposomes when compared with the association efficiency at $4^{\circ} \mathrm{C}$ (Figure 5B). Chlorpromazine $10 \mu \mathrm{g} / \mathrm{mL}$ also had a distinct effect on the cellular association of PF127-inlaid liposomes. As shown in Figure 5B, the total amount of cellassociated liposomes was markedly reduced to approximately $65 \%$, indicating that clathrin-mediated endocytosis was also involved in the cellular uptake of PF127-inlaid liposomes. For PF127-adsorbed liposomes, the mechanism was almost the same as that for unmodified liposomes. The cellular uptake decreased to $50 \%$ when incubated with filipin $1 \mu \mathrm{g} / \mathrm{mL}$, while other inhibitory agents showed no significant effect on the cellular uptake of PF127-adsorbed liposomes (Figure 5C).

\section{Discussion}

Modification of lipid systems by polymers may improve the efficiency of liposomal drug delivery systems by enhancing their physicochemical characteristics, eg, physical stability, encapsulation efficiency, bioadhesiveness, and "stealthing". A typical example is polyethylene glycol-conjugated lipid systems, which can "stealth" and stabilize liposomes, thereby prolonging the circulation half-life in vivo. Other polymers commonly used for modification of liposomal formulations are Poloxamers and Pluronics, a group of triblock copolymers with an amphiphilic nature. Different from PEGylated liposomes, the triblock copolymer in Pluronicmodified liposomes is either incorporated into or adsorbed onto the liposomal surface. This has been described by techniques such as nuclear magnetic resonance, differential scanning calorimetry, and dynamic light scattering. ${ }^{29,30}$ Further, it is well known that Pluronics, in aqueous solution, exist either as monomers or as self-assembled micelles. This behavior depends on the concentration and temperature of the surroundings. Hence when formulating the copolymer with liposomes, the Pluronics concentration and the temperature applied are two crucial factors determining the final composition and characteristics of the liposomes. In this study, PF127 

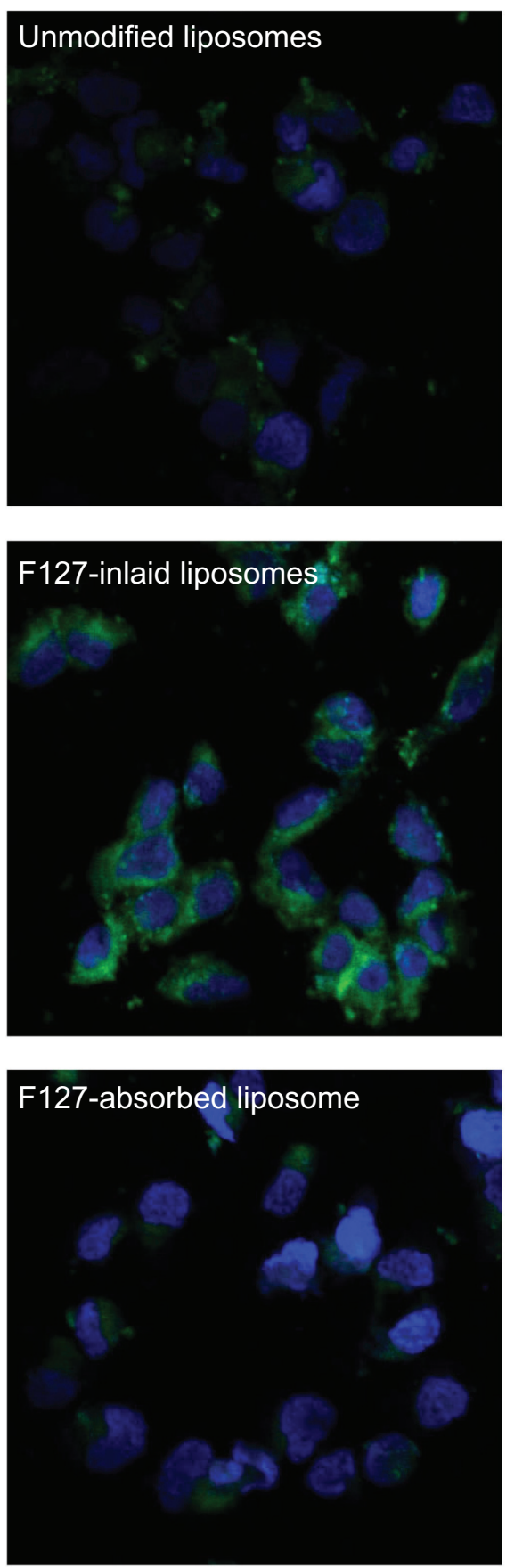
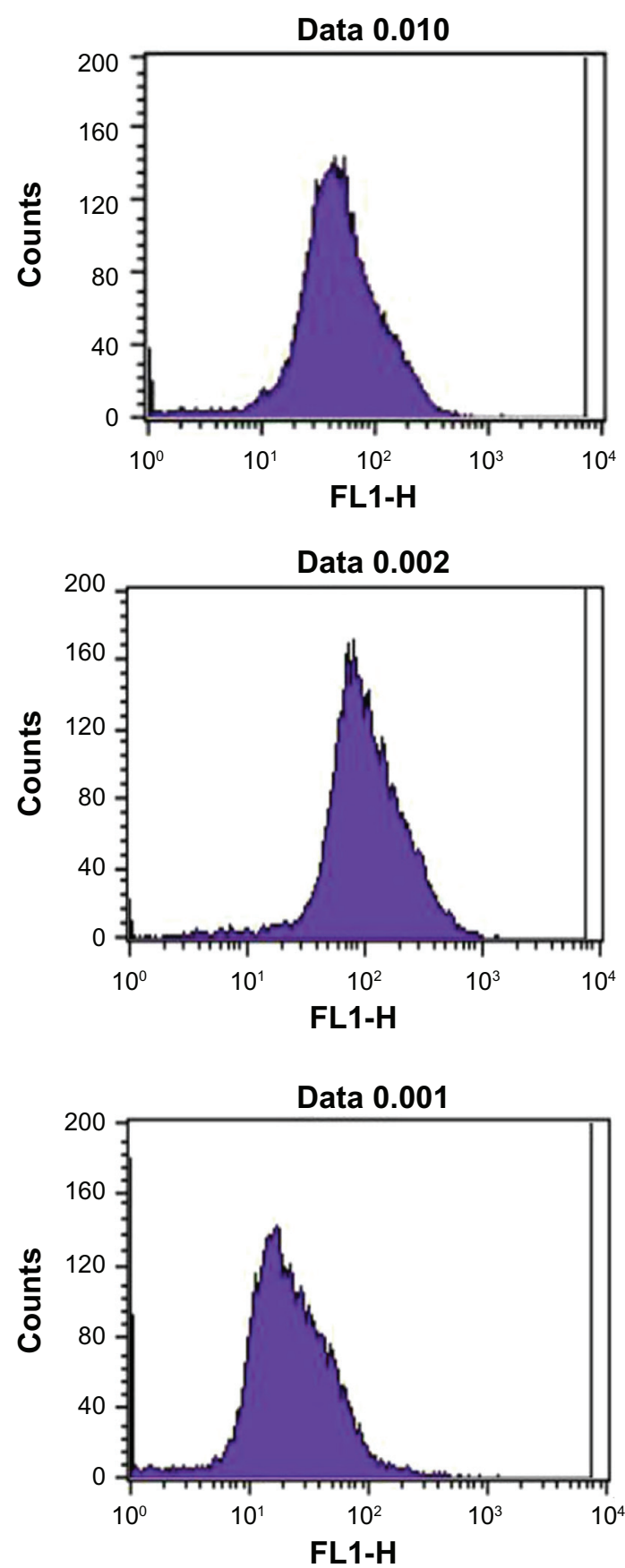

Figure 4 Confocal laser scanning microscopy and flow cytometry histogram of cellular uptake of coumarin 6-loaded liposomes, Pluronic ${ }^{\circledR}$ FI27-inlaid liposomes, and Pluronic FI27-adsorbed liposomes (green fluorescence) into Caco-2 cells. Nuclear staining was performed by DAPI (blue staining). The image was representative of three experiments with similar results.

was formulated with EPC liposomes at a concentration of $0.42 \%(\mathrm{w} / \mathrm{v})$. The preparation of PF127-modified liposomes were conducted using two methods at $25^{\circ} \mathrm{C}$. Because the concentration of $0.42 \%(\mathrm{w} / \mathrm{v})$ is below the critical micelle concentration of PF 127 at $25^{\circ} \mathrm{C}$, which is $0.7 \%(\mathrm{w} / \mathrm{v}),{ }^{31}$ PF127 are expected to be incorporated into the liposomes as monomers. This was confirmed by transmission electron microscopy and dynamic light scattering, both showing no formation of micelles. A schematic diagram of the PF127modified liposomes is shown in Figure 6, for PF127-inlaid liposomes, with PF127 actively involved in liposomal bilayer construction and distributed evenly across the bilayers, in contrast with PF127 absorbed liposomes, with PF127 absorbed solely on the surface of the liposome.

The detergent resistance experiment showed that PF127modified liposomes were stronger liposomal structures than 

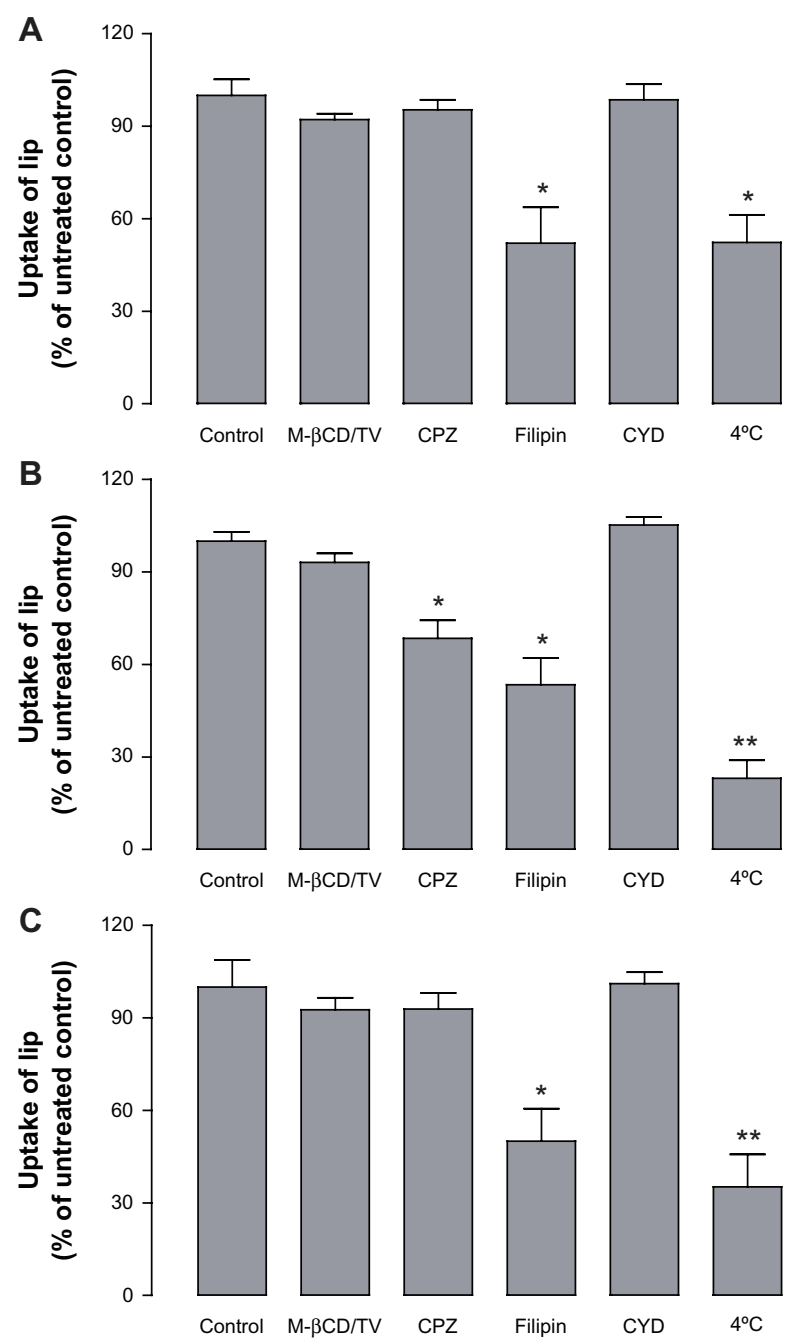

Figure $\mathbf{5}$ Effects of the different endocytotic inhibitors and temperature on the uptake of unmodified liposomes (A), Pluronic ${ }^{\circledR}$ FI27-inlaid liposomes (B), and Pluronic FI27-adsorbed liposomes (C) into Caco-2 cells.

Abbreviations: $M-\beta C D / T V$, methyl- $\beta$-cyclodextrin + lovastatin; $C P Z$, chlorpromazine; CYD, cyclochalasin D; lip, liposomal nanocarriers.

normal liposomes at the concentration of PF127 used in this study. These results indicate that PF127 modification could improve the stability of the liposome when used in vivo. PF127 stabilization possibly prevents the liposomal membrane from being distorted by surfactants, eg, the bile salts abundantly distributed in the gastrointestinal tract. Moreover, the improved rigidity of the liposomes may decrease drug leakage from nanocarriers, thus improving the stability of incorporated drugs. This would be of particular interest for enzyme-sensitive or acid-sensitive drug molecules.

\section{Mucus-penetrating properties of PFI27-modified liposomes}

Limited diffusion in intestinal mucus has been found to be one of the main limitations for drug delivery via the mucus membrane using nanocarriers like polymeric nanoparticles and liposomes. Our results indicate that the incorporation of PF127 into EPC liposomes facilitated their diffusion into rat intestinal mucus. Diffusion of unmodified liposomes through the intestinal mucus in the donor chamber of this experimental model was less than $10 \%$ within one hour, whereas the diffusion efficiency increased to $95 \%$ during the same time period across the polycarbonate membrane support alone. This suggests that diffusion of unmodified liposomes was restricted by native rat intestinal mucus. Further, it indicates that the modified Franz diffusion cell is a reliable model for evaluating the mucus-penetrating properties of nanocarriers.

Chen et al have reported that rat intestinal mucus is a barrier to diffusion of cyclosporin A-loaded liposomes. ${ }^{32}$ It was shown that most of the cyclosporin A-loaded liposomes were stranded in the rat intestinal mucus and unable to reach the intestinal wall. In our study, modification of liposomes with PF127 significantly enhanced diffusion efficiency through the native rat intestinal mucus. Compared with unmodified liposomes, the $\mathrm{P}_{\text {app }}$ of PF127-modified liposomes was increased by $5-7$-fold, and the percentage of cumulative amounts of coumarin 6 transported through the polycarbonate membrane was increased by six-fold. This increase might be due to the distribution of the hydrophilic polyoxyethylene part of PF127 on the surface of liposomes. This reduces the hydrophobic and electrostatic interactions of liposomal systems with mucin, allowing the nanocarriers to migrate through the mucus medium. Therefore, mucus clearance of the liposomes was minimized. However, inlaying or adsorbing the PF127 onto the liposomes did not seem to matter in terms of mucopenetration. This may due to the surface presence of polyoxyethylene chains of PF127, resulting in general hydrophilicity and shielding of surface charges.

\section{Caco- 2 cellular uptake}

Because a hydrophilic shell is usually biologically inert and will weaken the interaction of nanocarriers with a cell membrane, ${ }^{12,33}$ the cellular uptake of PF127-modified liposomes was investigated using Caco-2 cells. The results showed that, when loaded in PF127-inlaid liposomes, the cellular uptake of coumarin 6 was significantly increased as compared with unmodified liposomes. However, cellular uptake of coumarin 6 was decreased when it was formulated with PF127-absorbed liposomes. These results indicate that the cellular uptake efficiency of PF127-modified liposomes was directly affected by the modification method. The two modification methods resulted in a different incorporation pattern of PF127 in the liposomes. It has been reported that 


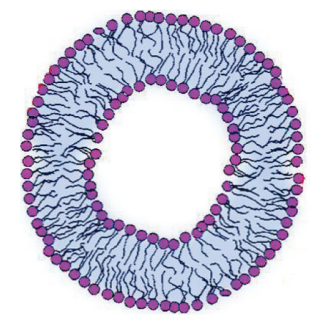

Unmodified liposomes

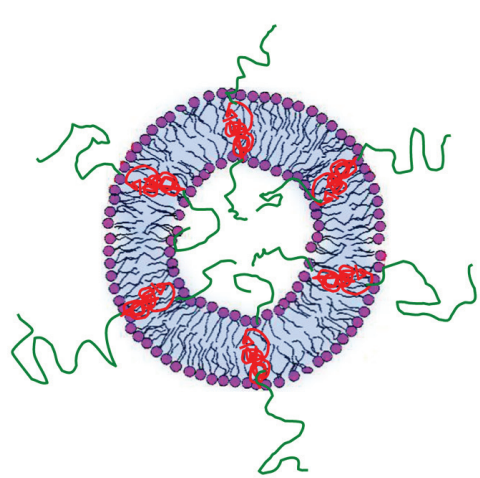

PF127-inlaid liposomes

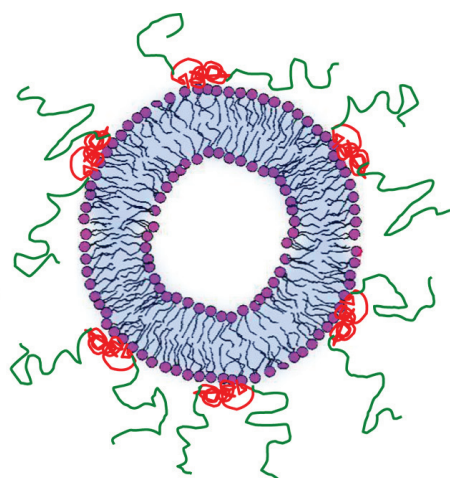

PF127-adsorbed liposomes

Figure 6 Schematic presentation of different liposomal nanocarriers. Green represents the polyethylene oxide chain of Pluronic ${ }^{\circledR}$ FI27; red represents the polyoxypropylene chain of Pluronic FI27.

addition of PF127 during vesicle formation (PF127-inlaid liposomes) resulted in polymer incorporation in both the inner and outer leaflets of the lipid bilayer, whereas all PF127 was associated only with the outer leaflet of the bilayer when PF127 was added after vesicle formation (PF127-absorbed liposomes) as illustrated in Figure 6. ${ }^{19}$

It is widely accepted that Pluronic polymers can stabilize and seal damaged lipid bilayer membranes. ${ }^{34-36}$ Considering that the PF127 is adsorbed on the outer leaflet of the bilayer (PF127-adsorbed liposomes), it may be easily lost by simple diffusion. It might then interact with the cell membrane, altering the membrane fluidity and membrane interaction, and finally stabilizing the membrane structure. Hence the cellular uptake of liposomes was decreased. This is strongly supported by the fact that the cellular uptake mechanism of PF127-adsorbed liposomes was quite similar to that of the unmodified liposomes. Sarah et $\mathrm{al}^{37}$ have reported both impaired Caco-2 cell viability and monolayer integrity when Pluronic F68 was used to deliver poorly soluble drugs.

However, for PF127-inlaid liposomes, when PF127 was inserted into both the inner and outer leaflet of the bilayer, the interaction between PF127 and the vesicles was much stronger. Thus, dissociation of PF127 from liposomes is unlikely to happen. As for the mechanism of cellular uptake of PF127inlaid liposomes, in addition to the caveolae-mediated uptake pathway, clathrin-mediated endocytosis had been introduced, as shown in the Caco-2 cell study as well. This mechanism of cellular uptake for PF127-inlaid liposomes is similar to that of Pluronic micelles. It has been reported that Pluronic P85 micelles were internalized by Caco-2 cells via clathrinmediated and caveolae-mediated endocytosis. ${ }^{38} \mathrm{~A}$ significant reduction in Caco-2 cell association efficiency at $4^{\circ} \mathrm{C}$ was observed in both PF127-inlaid liposomes and PF127-absorbed liposomes as compared with that at $37^{\circ} \mathrm{C}$. For PF127-adsorbed liposomes, this may due to aggregation at $4^{\circ} \mathrm{C}$. Because the particle size change for PF127-inlaid liposomes was less obvious than for PF127-adsorbed liposomes, the cellular uptake efficiency of PF127-inlaid liposomes should be less affected by low temperature. However the results showed that cellular uptake was more inhibited by low temperature, which again confirmed that cellular uptake of PF127-inlaid liposomes relied more on an energy-dependent pathway.

Most of the recent studies of polymer-modified nanocarriers were conducted by simply incubating nanocarriers with a polymer solution to enable absorption of the polymer onto the surface of the nanocarriers. ${ }^{39-41}$ Our studies clearly demonstrate that different modification methods result in different liposomal constructs with different cellular uptake efficiency.

\section{Conclusion}

Two types of mucus-penetrating liposomal formulations, ie, PF127-laid liposomes and PF127-adsorbed liposomes, were designed by incorporating PF127 with EPC lipids prior to or after vesicle formation. In vitro mucus-penetrating studies showed that the two types of PF127-modified liposomes possessed similar mucus-penetrating efficiency, and PF127 could significantly improve the mucus-penetrating properties of EPC liposomes in native rat intestinal mucus. A modified Franz diffusion cell model was established, which was demonstrated to be useful when studying mucus-penetrating properties of nanocarrier systems, eg, liposomes. In Caco-2 cellular uptake studies, PF127-inlaid liposomes exhibited higher cellular uptake efficiency when compared with PF127adsorbed liposomes as well as unmodified liposomes. The distinct cellular uptake mechanisms of PF127-inlaid liposomes from PF127-adsorbed liposomes were most likely due to the different constructs of the two types of PF127-modified lipo- 
somes produced. Taking both mucus-penetrating properties and cellular uptake into account, PF127-inlaid liposomes were shown to be very promising in drug delivery via mucus membranes of different cavities, eg, the intestine.

\section{Acknowledgment}

This work was supported by the Novo Nordisk-Chinese Academy of Science Research Foundation (NNCAS-2009-10) and National Science and Technology Major Project Key New Drug Creation and Manufacturing Program (2009ZX09301001). The work was also supported in part by the National Basic Research Program of China (2009CB930300).

\section{Disclosure}

The authors report no conflicts of interest in this work.

\section{References}

1. Fricker G, Kromp T, Wendel A, et al. Phospholipids and lipid-based formulations in oral drug delivery. Pharm Res. 2010;27(8):1469-1486.

2. Guan P, LuY, Qi J, et al. Enhanced oral bioavailability of cyclosporine A by liposomes containing a bile salt. Int J Nanomedicine. 2011;6: 965-974.

3. Irie T, Watarai S, Kodama H. Humoral immune response of carp (Cyprinus carpio) induced by oral immunization with liposomeentrapped antigen. Dev Comp Immunol. 2003;27(5):413-421.

4. Niu MM, Lu Y, Hovgaard L, Wu W. Liposomes containing glycocholate as potential oral insulin delivery systems: preparation, in vitro characterization, and improved protection against enzymatic degradation. Int J Nanomedicine. 2011;6:1155-1166.

5. Mishra B, Shukla D, Chakraborty S, Singh S. Lipid-based oral multiparticulate formulations - advantages, technological advances and industrial applications. Expert Opin Drug Deliv. 2011;8(2):207-224.

6. Galindo-Rodriguez SA, Allemann E, Fessi H, Doelker E. Polymeric nanoparticles for oral delivery of drugs and vaccines: a critical evaluation of in vivo studies. Crit Rev Ther Drug Carrier Syst. 2005;22(5): 419-464.

7. Ponchel G, Irache J. Specific and non-specific bioadhesive particulate systems for oral delivery to the gastrointestinal tract. Adv Drug Deliv Rev. 1998;34(2-3):191-219.

8. Atuma C, Strugala V, Allen A, Holm L. The adherent gastrointestinal mucus gel layer: thickness and physical state in vivo. Am J Physiol Gastrointest Liver Physiol. 2001;280(5):G922-G929.

9. Lai SK, Wang YY, Hanes J. Mucus-penetrating nanoparticles for drug and gene delivery to mucosal tissues. Adv Drug Deliv Rev. 2009;61(2): 158-171.

10. Dawson M, Wirtz D, Hanes J. Enhanced viscoelasticity of human cystic fibrotic sputum correlates with increasing microheterogeneity in particle transport. J Biol Chem. 2003;278(50):50393-50401.

11. Norris DA, Sinko PJ. Effect of size, surface charge, and hydrophobicity on the translocation of polystyrene microspheres through gastrointestinal mucin. J Appl Polym Sci. 1997;63(11):1481-1492.

12. Roger E, Lagarce F, Garcion E, Benoit JP. Biopharmaceutical parameters to consider in order to alter the fate of nanocarriers after oral delivery. Nanomedicine (Lond). 2010;5(2):287-306.

13. Romero EL, Morilla MJ. Topical and mucosal liposomes for vaccine delivery. Wiley Interdiscip Rev Nanomed Nanobiotechnol. 2011;3(4): 356-375.

14. Min T, Gorde A, Bodas M, Vij N. Mucus penetrating targeted nano-drug delivery system for cystic fibrosis. Pediatr Pulmonol. 2010;45:305. http://onlinelibrary.wiley.com/doi/10.1002/ppul.21339/abstract. Accessed November 25, 2011.
15. Yang M, Lai SK, Wang YY, et al. Biodegradable nanoparticles composed entirely of safe materials that rapidly penetrate human mucus. Angew Chem Int Ed Engl. 2010;50(11):2597-2600.

16. $\mathrm{Cu}$ Y, Saltzman WM. Controlled surface modification with poly(ethylene)glycol enhances diffusion of PLGA nanoparticles in human cervical mucus. Mol Pharm. 2009;6(1):173-181.

17. Suk JS, Lai SK, Wang YY, et al. The penetration of fresh undiluted sputum expectorated by cystic fibrosis patients by non-adhesive polymer nanoparticles. Biomaterials. 2009;30(13):2591-2597.

18. Hanes J, Suk JS, Lai SK, Boylan NJ, Dawson MR, Boyle MP. Rapid transport of muco-inert nanoparticles in cystic fibrosis sputum treated with N-acetyl cysteine. Nanomedicine. 2011;6(2):365-375.

19. $\mathrm{Wu} \mathrm{G}$, Lee KY. Interaction of poloxamers with liposomes: an isothermal titration calorimetry study. J Phys Chem B. 2009;113(47): 15522-15531.

20. Wei SJ, Smith NB. Improvements in the preparation of liposomes for electron-microscopy and electron-spectroscopic imaging (ESI). Microsc Res Tech. 1994;27(6):557-558.

21. Carrier RL, Crater JS. Barrier properties of gastrointestinal mucus to nanoparticle transport. Macromol Biosci. 2010;10(12):1473-1483.

22. Moghaddam FA, Atyabi F, Dinarvand R. Preparation and in vitro evaluation of mucoadhesion and permeation enhancement of thiolated chitosan-pHEMA core-shell nanoparticles. Nanomedicine. 2009;5(2): 208-215.

23. Sanders NN, De Smedt SC, Van Rompaey E, Simoens P, De Baets F, Demeester J. Cystic fibrosis sputum: a barrier to the transport of nanospheres. Am J Respir Crit Care Med. 2000;162(5):1905-1911.

24. Garcion E, Lamprecht A, Heurtault B, et al. A new generation of anticancer, drug-loaded, colloidal vectors reverses multidrug resistance in glioma and reduces tumor progression in rats. Mol Cancer Ther. 2006;5(7):1710-1722.

25. Takeuchi H, Makhlof A, Fujimoto S, Tozuka Y. In vitro and in vivo evaluation of WGA-carbopol modified liposomes as carriers for oral peptide delivery. Eur J Pharm Biopharm. 2011;77(2):216-224.

26. McClean S, Prosser E, Meehan E, et al. Binding and uptake of biodegradable poly-DL-lactide micro- and nanoparticles in intestinal epithelia. Eur J Pharm Sci. 1998;6(2):153-163.

27. Zhou Y, Hancock JF, Lichtenberger LM. The nonsteroidal anti-inflammatory drug indomethacin induces heterogeneity in lipid membranes: potential implication for its diverse biological action. PLoS One. 2010;5(1):e8811.

28. Huth US, Schubert R, Peschka-Suss R. Investigating the uptake and intracellular fate of $\mathrm{pH}$-sensitive liposomes by flow cytometry and spectral bio-imaging. J Control Release. 2006;110(3):490-504.

29. Feitosa E, Winnik FM. Interaction between Pluronic F127 and dioctadecyldimethylammonium bromide (DODAB) vesicles studied by differential scanning calorimetry. Langmuir. 2010;26(23):17852-17857.

30. Kostarelos K, Tadros TF, Luckham PF. Physical conjugation of (tri-) block copolymers to liposomes toward the construction of sterically stabilized vesicle systems. Langmuir. 1999;15(2):369-376.

31. Chieng YY, Chen SB. Interaction and complexation of phospholipid vesicles and triblock copolymers. J Phys Chem B. 2009;113(45):14934-14942

32. Chen Y, Ping QN, Guo JX, Lv WL, Gao J. The absorption behavior of cyclosporin A lecithin vesicles in rat intestinal tissue. Int J Pharm. 2003;261(1-2):21-26.

33. Chandaroy P, Sen A, Alexandridis P, Hui SW. Utilizing temperaturesensitive association of Pluronic F-127 with lipid bilayers to control liposome-cell adhesion. Biochim Biophys Acta. 2002;1559(1):32-42.

34. Maskarinec SA, Hannig J, Lee RC, Lee KYC. Direct observation of Poloxamer 188 insertion into lipid monolayers. Biophys $J$. 2002;82(3):1453-1459.

35. Chang LC, Lin CY, Kuo MW, Gau CS. Interactions of Pluronics with phospholipid monolayers at the air-water interface. J Colloid Interface Sci. 2005;285(2):640-652.

36. Maskarinec SA, Wu GH, Lee KYC. Membrane sealing by polymers Ann N Y Acad Sci. 2005;1066:310-320. 
37. Fischer SM, Brandl M, Fricker G. Effect of the non-ionic surfactant Poloxamer 188 on passive permeability of poorly soluble drugs across Caco-2 cell monolayers. Eur J Pharm Biopharm.79(2):416-422.

38. Sahay G, Batrakova EV, Kabanov AV. Different internalization pathways of polymeric micelles and unimers and their effects on vesicular transport. Bioconjug Chem. 2008;19(10):2023-2029.

39. Santander-Ortega MJ, Csaba N, Alonso MJ, Ortega-Vinuesa JL, BastosGonzalez D. Stability and physicochemical characteristics of PLGA, PLGA: poloxamer and PLGA: poloxamine blend nanoparticles - a comparative study. Colloids and Surfaces A Physicochemical and Engineering Aspects. 2007;296(1-3):132-140.
40. Xing JF, Deng LD, Dong AJ. Chitosan/alginate nanoparticles stabilized by Poloxamer for the controlled release of 5-fluorouracil. J Appl Polym Sci. 2010;117(4):2354-2359.

41. Luo GP, Jin C, Long J, et al. RNA interference of MBD1 in BxPC-3 human pancreatic cancer cells delivered by PLGA-Poloxamer nanoparticles. Cancer Biol Ther. 2009;8(7):594-598.

\section{Publish your work in this journal}

The International Journal of Nanomedicine is an international, peerreviewed journal focusing on the application of nanotechnology in diagnostics, therapeutics, and drug delivery systems throughout the biomedical field. This journal is indexed on PubMed Central, MedLine, CAS, SciSearch ${ }^{\circledR}$, Current Contents ${ }^{\circledR} /$ Clinical Medicine,
Journal Citation Reports/Science Edition, EMBase, Scopus and the Elsevier Bibliographic databases. The manuscript management system is completely online and includes a very quick and fair peer-review system, which is all easy to use. Visit http://www.dovepress.com/ testimonials.php to read real quotes from published authors. 